\title{
Ethical considerations in uterus transplantation
}

This article was published in the following Dove Press journal:

Medicolegal and Bioethics

30 September 2015

Number of times this article has been viewed

\author{
Jessica R Woessner' \\ Valarie K Blake ${ }^{2}$ \\ Kavita Shah Arora ${ }^{1,3}$ \\ 'Department of Obstetrics and \\ Gynecology, MetroHealth Medical \\ Center, Cleveland, OH, USA; ${ }^{2}$ College \\ of Law, West Virginia University, \\ Morgantown, WV, USA; ${ }^{3}$ Department \\ of Bioethics, Case Western Reserve \\ University, Cleveland, $\mathrm{OH}$, USA
}

\begin{abstract}
Uterus transplantation offers a novel treatment option for women with uterine factor infertility. While currently in the early clinical research phase, uterus transplantation will likely provide an alternative to adoption or gestational surrogacy, which is not permitted in some parts of the world. It uniquely allows for women with uterine factor infertility to experience the gestational component of motherhood and also to potentially share a genetic link with the offspring. Clinical research on uterus transplantation has been in process since the 1960s, and the first human live birth following transplant was reported in 2014. However, given the rapid clinical advancement, it is important to review the medical safety of the procedure and ethical dilemmas for the donor, recipient, and resulting child as well as the regulatory landscape that will ultimately guide clinical adoption. Given that uterus transplantation brings together the challenges of both assisted reproductive technology as well as organ transplantation as the world's first ephemeral transplant, it is important to discuss the ethical, legal, and social implications prior to moving from research to widespread clinical practice.
\end{abstract}

Keywords: ethics, regulation, infertility

\section{Ethical considerations in uterus transplantation}

Organ transplantation has been performed since the early 20 th century for a variety of reasons, including restoring function, saving life, and extending lives. Organ transplantation raises its own ethical considerations, including the use of cadaveric or living donors, allocation of scarce resources, use of medical and other criteria to determine eligibility, and informed consent. ${ }^{1-3}$ Improving the quality of a patient's life, rather than saving life itself, is a more recent goal and achievement of organ transplant (eg, hand, corneal, and face transplants) that presents further ethical considerations. ${ }^{1,3}$ For example, it is unclear how much risk is justified in the face of interventions aimed at improving quality of life rather than saving life. Uterus transplantation raises these same issues along with novel ones. For example, it is the first organ transplant with the goal being reproduction. Also, uterus transplantation is the first ephemeral organ transplant, or a transplant designed specifically for a short term, rather than the anticipated long-term nature of a transplanted liver or kidney, for example.

As a transplant with reproduction as its goal, it necessarily raises ethical questions about technological aid of reproduction, including the rights of any reproductive donors, the interests of the child, commodification of women's bodies, the interests and goals of the mother, and the role of the state in regulating women's bodies and reproduction. ${ }^{1}$ To women with uterine factor infertility (UFI) or infertility due to an anatomic issue specifically surrounding the uterus, uterus transplantation offers a novel 
treatment approach in addition to the currently available options of adoption and gestational surrogacy. ${ }^{4}$ However, transplantation for the goal of reproduction steps beyond the ethical debate in both assisted reproduction and transplantation, making it important to analyze the ethical, legal, and social implications of uterus transplantation prior to moving from the research phase to clinical practice.

\section{A novel reproductive alternative}

Infertility affected over 48 million couples around the world in 2010. ${ }^{5}$ Many factors lead to a diagnosis of infertility. In approximately one-third of infertility cases, the cause of the infertility is due to a factor involving the male, such as a low sperm count or motility. Additionally, one-third of infertility cases are due to factors surrounding the female whether due to decreased egg quality, difficulties with ovulation, or anatomic abnormalities that impact the normal trajectory of the egg or fertilized embryo from the fallopian tubes to implantation in the uterus. Finally, one-third of infertility cases are due to factors involving both partners or an unidentifiable cause. ${ }^{6}$ The specific anatomic abnormality of UFI affects approximately $3 \%$ of all infertile women, or approximately 9.5 million out of the 62 million women of reproductive age in the United States. ${ }^{1,7}$ UFI can be present due to congenital, disease-related, or iatrogenic causes. For example, a woman can be born without a uterus (Mayer-Rokitansky-KusterHauser syndrome), the uterus may have been removed as a component of surgical staging and treatment for cervical cancer, or an emergency hysterectomy may have been completed during a postpartum hemorrhage after delivery or after a trauma. ${ }^{1,8-10}$ Prior to uterus transplantation, patients with UFI did not have clinical interventions available that made pregnancy possible, leaving surrogacy and adoption as the only options for family-building.

While both adoption and gestational surrogacy allow women to parent a child, adoption does not allow for a genetic link to the future child, and neither option allows for the experience of the gestational component of motherhood. Furthermore, in many countries, surrogacy is legally banned (France and Italy for altruistic surrogacy, and Australia, France, Italy, New Zealand, South Africa, and the UK for commercial surrogacy). Some parts of the world discourage surrogacy for religious reasons or place bans on surrogacy contracts or surrogacy brokerage (eg, Hong Kong). ${ }^{1,11}$ Women with UFI in these places do not have any opportunities to have a genetic link to their children as the only currently available option is adoption. ${ }^{1,11}$ Further discussion surrounding the ethics of surrogacy and/or adop- tion versus uterus transplantation follows in the "Ethical implications" section.

The appeal of uterus transplantation as a needed alternative to surrogacy and adoption is clear, and as its safety progresses, it will likely face increasing demand from women with UFI. For example, in one clinical trial, over 500 women signed up to be experimental participants before this procedure had even been successfully demonstrated in humans. ${ }^{1,12}$

Uterus transplantation presents new and old ethical challenges to both transplant medicine as it is ephemeral and performed for quality-of-life reasons as well as in the world of assisted reproductive technology given the medical complexity, need for a donor, and risks to the future child. No existing transplants present ideal ethical models for uterus transplantation because the risk is distinct from other transplants in quality and time. Current transplantation procedures on other organ systems are generally lifesaving or -extending, making their benefit to the patient intuitive. With uterus transplantation, however, the risk versus benefit calculus is less straightforward, as the organ neither saves nor prolongs life, but is instead transplanted for quality-of-life purposes. To obviate the need for long-term immunosuppressive medications, the transplanted uterus would be removed after childbearing is complete. The need to perform at least two (transplant and removal) major abdominal surgeries thus adds to the risk profile of uterus transplantation compared to other organs transplanted for quality-of-life purposes.

Uterus transplantation also stands apart in that the major intent is for procreation, which introduces a further layer of ethical complexity. Ovarian tissue has been successfully transplanted since the year 2004, with the first documented case being a woman with non-Hodgkin's lymphoma who had her ovarian tissue removed and frozen prior to starting chemotherapy. ${ }^{1,13}$ Following completion of her cancer treatment, her own ovarian tissue was transplanted into her body, resulting in successful pregnancy and a live birth. There have also been documented cases of ovarian tissue being transplanted both between monozygotic twins and in nonidentical siblings after bone marrow transplantation resulting in complete chimerism. ${ }^{14-16}$ Another example of a successfully transplanted reproductive organ is the testicle. The first documented case of this is from 2001, when a man had his testicular tissue removed prior to cancer treatment and re-transplanted following chemotherapy and successfully fathered a live birth. ${ }^{14,17}$ Both of these ethically and legally complex technologies are also only in research phases. While uterus transplantation represents a novel combination 
of transplant and reproductive medicine, the scientific and clinical interest in uterus transplantation is not new.

\section{Review of the scientific research}

While research on uterus transplantation has been conducted for over 50 years, in 2014, a Swedish team announced the first human birth after uterus transplantation. Born prematurely due to preeclampsia and via cesarean section, this remarkable live birth represents a pivotal success after decades of research. ${ }^{18}$ The early path to uterus transplantation began in the 1960s with research on canine models. ${ }^{7}$ There was little forward progress during the 1970s-1990s, given the focus on other assisted reproductive technologies such as in vitro fertilization and intracytoplasmic sperm injection. Research on uterus transplantation again emerged in the early 2000s as early studies from El-Akouri et al in Sweden demonstrated successful fertility in the mouse model following syngeneic uterus transplants. ${ }^{19}$ Allogenic uterus transplant was demonstrated in mice in 2006 and rats in $2010 .^{7}$ Avison et al were subsequently able to confirm long-term viability of the uterus after transplant in the miniature swine model in $2009 .{ }^{20}$ Then, in 2011, Ramirez et al transplanted a uterus in a sheep and demonstrated successful fertility following transplantation. ${ }^{21}$ While these early experimental animal models demonstrated the feasibility of uterus transplantation, more preclinical research was necessary before moving to human models.

The first report of uterus transplantation in a nonhuman primate was by Johannesson et al in 2013. In this study, Johannesson et al looked at not only the feasibility of uterus transplantation, but also at the immunosuppressive therapy required to suppress transplant rejection in the recipient. This study included 18 female baboon donors and recipients who underwent a hysterectomy; bilateral salpingo-oophorectomy; and bilateral uterine, internal iliac artery, and ovarian vein transplantation. The ovaries were transplanted in order to evaluate the graft by monitoring hormonal cyclicity. The transplant recipients were then divided into three groups who received either no immunosuppressive therapy, monotherapy, or induction immunotherapy with triple therapy. All baboons survived the surgical procedures, with $40 \%$ of transplant recipients resuming hormonal cyclicity. However, all recipients experienced some level of transplant rejection. ${ }^{22}$ This study was the first to show the feasibility of live uterus transplantation in primates, though a satisfactory immunosuppressive regimen had not yet been demonstrated.

The preclinical research was continued by Saso et al, who in 2014 reported their experience with uterus transplantation in rabbits using a macrovascular patch technique and postoperative tacrolimus for immunosuppression to evaluate long-term uterine graft survival. The macrovascular patch technique was attempted given the difficulty in achieving an adequate blood supply for the graft once transplanted in the recipient. This study involved five allogenic white rabbit uterine cross-transplants in rabbits with previously proven fertility. While all five recipients survived the initial surgery, three died by postoperative day 4 , and neither survivor successfully conceived. Necropsy at 9 and 11 months showed a lack of patency of uterine cornua at the point of anastomosis, leading the authors to conclude that, again, the feasibility of uterus transplantation was demonstrated; however, fertility was not. They also proposed that potential future studies could involve embryo transfer techniques to achieve conception given the lack of cornual patency. ${ }^{23}$

The first transplantation of a human uterus was attempted on April 6, 2000, in Saudi Arabia. The transplant was performed on a 26-year-old female who had required a hysterectomy in 1994 after suffering from a postpartum hemorrhage at the time of a cesarean delivery. The donor uterus was from a 46-yearold woman. The transplant recipient experienced mild acute withdrawal on postoperative day 9 with low-grade fevers but was successfully treated. She was started on hormonal therapy and successfully experienced two withdrawal bleeds. On the 99th day postoperatively, she experienced foul-smelling vaginal discharge, and examination revealed uterine prolapse, with ultrasound confirming acute vascular thrombosis. She therefore underwent hysterectomy. Although fertility of the transplanted organ had not been tested, this study demonstrated the feasibility of uterus transplantation in a human as well as acceptable shortand mid-term outcomes with one acute rejection episode which quickly resolved. ${ }^{24}$ The second attempt at uterus transplantation in a human took place in Turkey in 2011 when a young woman with Mayer-Rokitansky-Kuster-Hauser syndrome (congenital absence of the uterus and proximal vagina) was the recipient of a uterus donated by a multiorgan donor of approximately the same age. She began to menstruate regularly after 20 days with no rejection episodes. She subsequently underwent two rounds of embryo transfer (obtained via in vitro fertilization prior to transplant), though had two early miscarriages. No live births have been reported to date from this attempt. ${ }^{25}$

After the feasibility of human uterus transplantation was demonstrated in these studies, researchers attempted to move forward with clinical trials. Brännström et al conducted the first prospective clinical study on uterus transplantation in 2012-2013, consisting of a cohort of nine women at Sahlgrenska University Hospital in Gothenburg, Sweden. ${ }^{26}$ All participants in this study were confirmed to have UFI, 
eight of whom due to Mayer-Rokitansky-Kuster-Hauser syndrome and one having previously undergone a hysterectomy for a history of cervical cancer. Four of the donors were mothers of the patients, and all donors were postmenopausal. All recipients were kept on a standardized immunosuppression protocol involving both induction and maintenance therapy as well as antiviral prophylaxis. They were monitored twice weekly for the first month, once weekly during months 2-3, and every other week thereafter with both clinical evaluations as well as cervical biopsies, and ultrasound to evaluate blood flow to the uterus.

Postoperatively, two of the graft recipients required surgical removal due to complications. One patient on postoperative day 33 developed a positive Enterococcus faecalis cervical infection, which was initially treated successfully with intravenous antibiotics. She relapsed with infection several times, requiring multiple doses of IV antibiotics, hospitalizations, and drainage of abscesses, and eventually underwent hysterectomy on postoperative day 105 . Her graft revealed no evidence of rejection at time of surgery. The other patient requiring removal of her graft had sudden cessation of vascular flow by ultrasound on postoperative day 3 , with exam revealing a blood-congested cervix and surgical evaluation revealing focal necrosis and moderate ischemic myometrial damage but no evidence of graft rejection. The remaining seven patients retained viable uteri with resumption of menses during the 6-month follow-up period. ${ }^{26}$

The plan at the conclusion of the study was for the remaining seven patients to undergo embryo transfer 12-18 months after initial transplant if the clinical course remained uneventful, the first of which occurred in $2013 .{ }^{26}$ This live birth was reported in 2014 and was the first successful birth after uterus transplantation in a human. ${ }^{18,27}$ The patient was a 35-year-old woman with Mayer-Rokitansky-Kuster-Hauser syndrome and was the recipient of a uterus donated by her 61-year-old postmenopausal mother. Prior to the transplant procedure, the recipient underwent in vitro fertilization treatments with her partner, in which eleven embryos were cryopreserved. The donor was placed on 3 months of sequential oral contraceptive pills to ensure menstrual functionality of the uterus prior to the transplantation procedure. Both surgeries were uneventful, with good uterine perfusion noted at conclusion of transplantation. Both donor and recipient recovered completely and were discharged from the hospital on postoperative day $6 .{ }^{18}$

The recipient then underwent induction therapy with anti-thymocyte globulin as well as methylprednisolone, and her immunosuppression maintenance therapy consisted of oral tacrolimus and mycophenolate mofetil for 10 months followed by azathioprine to avoid the potentially teratogenic effects of mycophenolate. After 6 months, prednisolone was added to her maintenance therapy as she had undergone several mild rejection episodes, and she recovered well. Her first menstrual period occurred approximately 43 days posttransplant, followed by regular 32-day cycles. At 8 months and 12 days posttransplant, she was noted to have mild cervical dysplasia on her scheduled biopsy and underwent a small conization procedure. She then underwent embryo transfer 1 year following her transplant and had a normal gestational period until week 31 , at which time she developed preeclampsia and underwent a cesarean delivery. ${ }^{18,27}$ Both the patient and the infant recovered without issue following delivery. The patient continues to carry her transplanted uterus in hopes of a future, subsequent pregnancy and thus remains on an immunosuppressive regimen.

\section{Ethical implications}

Given that uterus transplantation is moving from the research phase to the clinical phase, it is important to review the ethical implications for all three parties involved - donor, recipient, and potential future child. While living donors were used in the studies by Brännström et al, ${ }^{18}$ both cadaveric and living donors remain a theoretical possibility and have been attempted in earlier research studies. Cadaveric donation may be of particular interest to some parts of the world where living organ donation is not permitted and in cases where individuals do not have surviving family members or friends willing to donate.

Given the presumption, based on rescue ethics, that organs harvested from deceased donors should only be used for lifesaving intervention, additional justification is needed for organs to improve quality rather than quantity of life. Rescue ethics, or the Rule of Rescue, has been applied in bioethics in numerous contexts. However, in this context, rescue ethics states that physicians, medicine in general, and society at large have an obligation to save "endangered life when possible". ${ }^{28}$ Thus, organs from donors should be given priority if they can be used for a lifesaving indication, such as heart or lung transplants. Those that are not lifesaving, such as the uterus, should therefore assume lower societal priority and require additional justification to complete. Previous transplant teams undertaking face, cornea, or hand transplants have utilized additional consent procedures apart from the typical organ donation consent processes. ${ }^{1,29}$ In this manner, consent from the deceased person's surrogate(s) is obtained separate from and after, consent is obtained for the transplant of vital, 
lifesaving organs. However, given the public's lack of familiarity with uterus transplantation and the unique ethical and legal considerations surrounding reproductive decision-making, surrogate consent is not now and may never be appropriate. Transplant of the uterus should ideally occur only from donors who expressed such a desire when living, and further research should be undertaken to understand this unique issue of cadaver donation of reproductive material, particularly with respect to laws surrounding surrogate consent for cadaver donation.

If utilizing living donors, the concern surrounding medical safety is paramount. While hysterectomies are performed daily around the world for many reasons, hysterectomies are not without risk to the patient, including major blood loss, injury to bowel or bladder, thrombosis, adverse anesthetic reactions, and death. ${ }^{30}$ While these hysterectomies are medically indicated for a variety of reasons and thus offer benefit to the patient, there is no such medical benefit to a hysterectomy to serve as a uterus donor. Furthermore, due to the need to preserve vascular pedicles, the length of procurement surgery reported by Brännström et al was 10 hours and 7 minutes, which far exceeds the typical length of surgery for most hysterectomies for clinical indications. ${ }^{1,18,31}$

Adding to the medical risk is the concern regarding consent free of coercion. Given that most donors in the study by Brännström et $\mathrm{al}^{18}$ were family members of the recipients, promoting voluntary consent is challenging. The transplant team should ideally be divided between those caring for the donor and those for the recipient, with an independent donor advocate being assigned to protect the best interests of the prospective donor as in other living donor situations. Donors should be tested for both medical and psychological suitability, understand the risks and benefits for both herself and the recipient, and know the potential impact on future health and insurance coverage. Donor advocates, which are legally required in other living donor contexts, also protect the right of the donor to opt out at any stage of the process without the recipient knowing why. ${ }^{1,32,33}$ Utilizing family members as donors thus adds layers of complexity to the standard transplant informed consent process, though is a situation that is relatively common in transplant medicine.

There are also several concerns for recipients of transplanted uteri, which far outweigh the risks posed by previous assisted reproductive technologies. After the initial transplant surgery, reported to have required 4 hours and 55 minutes by Brännström et al, at least two additional major surgeries would be required in the event if the transplant is successful and the recipient is able to carry a pregnancy: a cesarean section for delivery and a postpartum hysterectomy to prevent necessity for lifelong immunosuppressive therapy. ${ }^{18}$ The purpose of this medical risk is to experience the gestational component of motherhood, and possibly the genetic if gestational surrogacy is not available. Yet, given the inability to anastomose nerves, it is unclear how the sensation of pregnancy will vary for patients of uterus transplantation versus women who naturally conceive. For example, while the patient will experience morning sickness and experience swelling and an enlarging uterus, it is unclear whether the sensation of contractions or fetal movement will be the same. Thus, the experience of pregnancy, the very goal of uterus transplantation versus surrogacy or adoption, will differ for recipients than for those who spontaneously conceive and carry a pregnancy. It is also important for obstetricians to be aware that these anticipated sensations may differ from those of women who spontaneously conceive, and thus differing labor precautions may be needed for transplant patients.

Consent is also crucial for potential recipients and patients of uterus transplantation. In a research context, consent must be voluntary, explain the experimental nature of the procedure, review benefits to the participant versus others through increased knowledge, discuss alternatives, and comprehensively discuss risks. Given the psychology of infertility, the potential for therapeutic misconception in the early research phases is clear. ${ }^{1,34}$ It is important for the transplant team to be clear that, as of yet, uterus transplantation remains research and that guarantee of outcome (ie, a child) cannot be made. As research continues, the results of these studies should continue to guide and inform future research direction and the consent process for future patients; for example, a careful examination of how these patients experience pregnancy so that future patients can consider this in their risk/benefit analysis and their goals for the procedure.

Furthermore, complexity surrounding an exit strategy is more difficult for uterus transplantation than for other organs. Given the possibility of organ rejection during pregnancy, both the physician as well as the organ recipient would need to decide if and when to terminate the pregnancy in an effort to potentially save the patient from severe organ rejection. ${ }^{1}$ The emotional difficulty of this situation is further complicated by legal and religious limitations on terminations in many countries, including conscience-based policies and gestational age limitations. Ideally, this conversation would occur during the initial informed consent process prior to organ transplantation. ${ }^{27}$

Unique to uterus transplantation versus other transplanted organs is the need to consider the well-being of the future intended child, the goal of the transplant. Safety remains a key concern. While the immunosuppressive regimen that has been 
studied by Brännström et $\mathrm{al}^{18}$ is not teratogenic, high-quality data do not exist. Furthermore, there remains a risk of premature delivery and low birthweight, though it is unclear whether this is due to the mother's underlying medical comorbidities. ${ }^{1,3,25,35,36}$ Second, in the case of acute vascular thrombosis, it is unclear what the impact of hypoxia would be on the developing fetus. Due to these concerns, it is imperative that patients account for risks to the future child in their decision-making processes and that physicians recognize their double responsibility - both to the intended mother and to the future child. ${ }^{1}$

While these concerns are important, and point to the importance of thorough informed consent prior to uterus transplantation, it is important not to minimize infertility by being overly skeptical of treatment options. Studies in the United States demonstrate that infertile couples often feel their infertility is disregarded or minimized. ${ }^{1,34}$ Moreover, both adoption and surrogacy have risks and burdens as well, may be prohibited in some areas of the world, are fraught with their own ethical complexities, and do not allow for the fulfillment of the genetic and gestational components of motherhood. ${ }^{1}$ For example, uterus transplantation continues to share many of the ethical difficulties of other assisted reproductive technologies, such as questions surrounding the rights of any reproductive donors, the interests of the child, potential commodification of women's bodies, the interests and goals of the mother, and the role of the state in regulating women's bodies and reproduction. ${ }^{1}$ Specifically, compared to surrogacy and adoption as the currently available alternatives (where legal and available), uterus transplantation only exacerbates the issue of distributive justice, as the medical costs will be prohibitive to most unless covered by health insurance or subsidized by the government. The commodification concerns surrounding surrogacy are also only amplified, as the "desired" reproductive organs are simply moved from one body to another - thus, a woman is less a "vessel" and more simply a "pot holder". Given the state's interest in regulating various aspects of reproduction (whether from a quality and medical perspective, such as in in vitro fertilization, or a child's interest perspective, such as in adoption), the route through which uterus transplantation will be regulated remains to be seen. ${ }^{1}$

\section{Regulatory perspectives}

Apart from the medical challenges and ethical complexities surrounding uterus transplantation, there are many regulatory concerns as well. Uterus transplantation does not neatly fit into the realm of assisted reproductive technologies nor transplant medicine. There is some uncertainty, given reproductive and transplant aspects of uterus transplantation, about which bod- ies of law may regulate the procedure: the United Network for Organ Sharing (UNOS) or transplant regulations; US Food and Drug Administration regulations; ART laws, which mainly address success rates and very little other aspects of safety or availability; or the Uniform Anatomical Gift Act (which applies to most aspects of cadaver donation). ${ }^{37}$

From a financing and delivery perspective, in many countries, assisted reproductive technologies are distributed by the free market. Uterus transplantation, however, would be far more expensive than current infertility treatments and thus exacerbate the issue of distributive justice that already affects infertility care. Furthermore, many countries, including the United States under the Uniform Anatomical Gift Act, specifically prohibit the sale of organs, though the sale of reproductive tissue such as sperm and oocytes is permitted. ${ }^{1,2}$ While specific sale of the uterus may be prohibited in the United States, surrogacy, in one sense, already allows for the functional sale of the uterus and its products on the free market. The commodification issues surrounding egg donation and surrogacy, then, are only amplified by uterus transplantation. ${ }^{1,38}$

In the United States, if guided by the policies that govern organ transplantation, distribution would be guided by UNOS. At first glance, utilizing typical organ transplant distribution strategies obviates the concern of only the wealthiest women with UFI obtaining uterus transplants. However, UNOS allocates organs based on three basic principles: sickest first, prognosis of the recipient, and the first-come first-served principle. . $3,39,40$ As uterus transplants are not considered to be lifesaving nor -extending, these principles are difficult to apply. Prioritizing infertile women/couples and prognosis is difficult. It is unclear whether number of prior attempts, likelihood of success, prior living children, or impact of infertility on psychosocial well-being should be used to allocate uteri for transplant. ${ }^{1,14}$

Regulation surrounding transplant informed consent and organ donation practices remains complex and varies by country. The use of both cadaveric donors for non-vital organs or living, often related, donors is not typical for standard organ donation and justifies the use of additional and separate informed consent processes and donor advocates.

\section{Conclusion}

Over the last decade, there has been dramatic success with uterus transplantation rapidly moving from the preclinical to the clinical research stage. While the report of the first successful live birth following uterus transplantation in 2014 marked a potential new beginning for women and couples with UFI, there remain clinical, ethical, and regulatory challenges. The 
medical safety for the donor, recipient, and future child remains an area of active research interest. The ethical implications for all three parties should continue to be discussed, and the eventual uptake into clinical practice will require deliberation and modifications regarding regulatory process. However, as both adoption and gestational surrogacy do not potentially allow for a genetic and/or gestational link, uterus transplantation is a novel treatment option for women with UFI. Given this important clinical need, it is imperative that discussions surrounding the medical, ethical, legal, and social implications continue to guide the clinical science and implementation.

\section{Disclosure}

The authors report no conflicts of interest in this work.

\section{References}

1. Arora KS, Blake V. Uterus transplantation: ethical and regulatory challenges. J Med Ethics. 2014;40:396-400.

2. The National Conference of Commissioners on Uniform State Laws. Revised Uniform Anatomical Gift Act (2006). 2009. Available from: http://www.uniformlaws.org/shared/docs/anatomical_gift/uaga_final_ aug09.pdf. Accessed August 28, 2015.

3. Olausson M, Johannesson L, Brattgård D, et al. Ethics of uterus transplantations with live donors. Fertil Steril. 2014;102(1):40-43.

4. Shah K. Wombs for hire - the ethics of surrogacy. In: Goldfarb JM, editor. Third-Party Reproduction: A Comprehensive Guide. New York: Springer; 2014.

5. Mascarenhas MN, Flaxman SR, Boerma T, Vanderpoel S, Stevens GA. National, regional, and global trends in infertility prevalence since 1990: a systematic analysis of 277 health surveys. PLoS Med. 2012;9(12): e1001356.

6. Infertility fact sheet [webpage on the Internet]. Washington, DC: Office on Women's Health; [updated July 16, 2012]. Available from: http:/ www.womenshealth.gov/publications/our-publications/fact-sheet/ infertility.html. Accessed July 20, 2015.

7. Milliez J. Uterine transplantation FIGO Committee for the Ethical Aspects of Human Reproduction and Women's Health. Int J Gynaecol Obstet. 2009;106:270.

8. Altchek A. Uterus transplantation. Mt Sinai J Med. 2003;70(3): 154-162.

9. Pearson H. Infertility researchers target uterus transplant. Nature. 2007;445:466-467.

10. Nair A, Stega J, Smith JR, Del Priore G. Uterus transplant: evidence and ethics. Ann NY Acad Sci. 2008;1127:83-91.

11. Sreenivas K, Campo-Engelstein L. Domestic and international surrogacy laws: implications for cancer survivors. In: Woodruff TK, Zoloth L, Campo-Engelstein L, Rodriguez S, editors. Oncofertility: Ethical, Legal, Social, and Medical Perspectives. New York: Springer; 2010: 135-152.

12. Nair AR, Feret M, Del Priore G, Malanowska-Stega. Applicants for uterine transplantation: description of candidates. Fertil Steril. 2008;90:S170.

13. Donnez J, Dolmans MM, Demylle D, et al. Livebirth after orthotopic transplantation of cryopreserved ovarian tissue. Lancet. 2004;364(9443): 1405-1410.

14. Blake V, Shah K. Reproductive tissue transplants defy legal and ethical categorization. Virtual Mentor. 2012;14(3):232-236.

15. Silber SJ, Lenahan KM, Levine DJ, et al. Ovarian transplantation between monozygotic twins discordant for premature ovarian failure. N Engl J Med. 2005;353(1):58-63.
16. Donnez J, Squifflet J, Pirard C, Jadoul P, Dolmans MM. Restoration of ovarian function after allografting of ovarian cortex between genetically non-identical sisters. Hum Reprod. 2010;25(10):2489-2495.

17. Vince G. Man fathers child after testicular transplant. New Scientist. February 28, 2001. Available from: http://www.newscientist.com/article/ dn1851-man-fathers-child-after-testicular-transplant.html. Accessed April 10, 2015.

18. Brännström $M$, Johannesson $L$, Bokström $H$, et al. Livebirth after uterus transplantation. Lancet. 2015;385(9968):607-616.

19. El-Akouri R, Kurlberg G, Brännström M. Successful uterine transplantation in the mouse: pregnancy and post-natal development of offspring. Hum Reprod. 2003;18:2018-2023.

20. Avison DL, DeFaria W, Tryphonopoulos P, et al. Heterotopic uterus transplantation in a swine model. Transplantation. 2009;88:465-469.

21. Ramirez ER, Ramirez DK, Pillari VT, Vasquez H, Ramirez HA. Modified uterine transplant procedure in the sheep model. $J$ Minim Invasive Gynecol. 2008;15:311-314.

22. Johannesson L, Enskog A, Mölne J, et al. Preclinical report on allogeneic uterus transplantation in non-human primates. Hum Reprod. 2013;28(1): 189-198.

23. Saso S, Hurst $\mathrm{S}$, Chatterjee J, et al. Test of long-term uterine survival after allogeneic transplantation in rabbits. J Obstet Gynaecol Res. 2014; 40(3):754-762.

24. Fageeh W, Raffa H, Jabbad H, Marzouki A. Transplantation of the human uterus. Int J Gynaecol Obstet. 2002;76:245-251.

25. Johannesson L, Enskog A. Experimental uterus transplantation. Best Pract Res Clin Obstet Gynaecol. 2014;28(8):1198-1210.

26. Brännström M, Johannesson L, Dahm-Kähler P, et al. First clinical uterus transplantation trial: a six-month report. Fertil Steril. 2014; 101(5):1228-1236.

27. Arora KS, Blake V. Uterus transplantation: the ethics of moving the womb. Obstet Gynecol. 2015;125(4):971-974.

28. Bochner F, Martin ED, Burgess NG, Somogyi AA, Misan GMH. Controversies in treatment: how can hospitals ration drugs? Drug rationing in a teaching hospital: a method to assign priorities. BMJ. 1994;308:901.

29. Agich GJ. Extension of organ transplantation: some ethical considerations. Mt Sinai J Med. 2003;70(3):141-147.

30. Abdominal hysterectomy: Risks [webpage on the Internet]. Mayo Clinic; 2012. Available from: http://www.mayoclinic.com/health/ hysterectomy/MY00163/DSECTION=risks. Accessed April 6, 2015.

31. Keith LG, Del Priore G. Uterine transplantation in humans: a new frontier. Int J Gynaecol Obstet. 2002;76:243-244.

32. Advisory Committee on Organ Transplantation. Summary Recommendations to the Secretary [webpage on the Internet] Washington: US Department of Health and Human Services; 2012. Available from: http://organdonor.gov/legislation/acotsummaryrec. html. Accessed April 17, 2015.

33. Code of Federal Regulations, Title 42 - Public Health. Section 482.98 Condition of participation: Human resources. 2011(5). Available from Section 482.98 - Condition of participation: Human resources. Accessed September 7, 2015.

34. Orentlicher D. Toward acceptance of uterus transplants. Hastings Cent Rep. 2012;42(6):12-13.

35. Brännström M, Wranning CA, Marcickiewicz J, Enskog A, Hanafy A. Uterus transplantation - substantial progress in research but not yet ready for the clinic. Middle East Fertil Soc J. 2007;12:86-95.

36. Bahadur G. Death and conception. Hum Reprod. 2002;17(10): 2769-2775.

37. Blake VK. Ovaries, testicles, and uteruses, oh my! Regulating reproductive tissue transplants. William Mary J Women Law. 2013;19(2):353-393.

38. Steinbock B. Payment for egg donation and surrogacy. Mt Sinai J Med. 2004;71(4):255-265.

39. Persad G, Wertheimer A, Emanuel EJ. Principles for allocation of scarce medical interventions. Lancet. 2009;373:423-431.

40. Cozzoli M. Ethical issues of organ transplantation in non-life-saving situations. In: Lanzetta M, Dubernard JM, Petruzzo P, editors. Hand Transplantation. Milan: Springer Milan; 2007:111-114. 


\section{Publish your work in this journal}

Medicolegal and Bioethics is an international, peer-reviewed, open access journal exploring the application of law to medical and drug research and practice and the related ethical and moral considerations. The journal is characterized by the rapid reporting of reviews, case reports, guidelines and consensus statements, original research and surveys. The manuscript management system is completely online and includes a very quick and fair peer-review system. Visit http://www.dovepress.com/testimonials.php to read real quotes from published authors. 